\title{
Ultrasonic Evaluation of Common Carotid Intima-Media Thickness (IMT) - Influence of Local Plaque on the Relationship Between IMT and Age
}

\author{
Fujiko Ando, Kiyoshi Takekuma, Naoakira Niino, and Hiroshi Shimokata
}

\begin{abstract}
Ultrasonic evaluation of the intima-media thickness in the common carotid artery (IMT-CCA) has been widely used as a marker of atherosclerosis. However, the definition of IMT-CCA is not uniform and it includes two quite different pathological changes; a general intima-media thickening and a local atherosclerotic change (plaque formation). The aim of this paper was to evaluate the IMT-CCA and local atherosclerosis separately, and to clarify how the IMT-CCA itself changes with age and how local plaques influence the relationship between the IMT-CCA and age.

The subjects were 979 men and women aged 40 to 79 years who participated in the first wave examination of the National Institute for Longevity Sciences-Longitudinal Study of Aging (NILSLSA).

The IMT-CCA measured at the thinnest point was significantly higher in men $(0.61 \pm 0.15 \mathrm{~mm})$ than in women $(0.58 \pm 0.14 \mathrm{~mm}, p<0.01)$ and it increased with age (trend $p<0.0001)$ in both genders. The IMT-CCA was higher with the presence of plaque in the bulbs (PLQ-BLB) than without PLQ-BLB $(p<0.0001)$. Although this was a cross-sectional study, the IMT-CCA increased $0.06 \mathrm{~mm} / 10$ years with PLQ-BLB and $0.04 \mathrm{~mm} / 10$ years without PLQ-BLB. The IMT-CCA could be partially explained by age, gender and PLQ-BLB $\left(r^{2}=0.317\right)$.

In this middle-aged and elderly population, an increase in the IMT-CCA showed a moderate relationship with local atherosclerosis and age. J Epidemiol, 2000; 10 : S10-S17.
\end{abstract}

intima-media thickness (IMT), atherosclerosis, plaque, epidemiology, aging

\section{INTRODUCTION}

The quantitative assessment of atherosclerosis in populations is essential to a better understanding of the pathophysiology of the lesions and to the prediction or prevention of consequent development of atherosclerotic diseases. In recent years, ultrasonic measurement has been available for noninvasive assessment of local plaque formation and intima-media thickening in the extracranial carotid arteries ${ }^{1-8}$.

The local plaque formations in the internal or distal portion of the common carotid arteries (CCA) have been reported to be related to atherosclerotic diseases, such as ischemic heart disease ${ }^{9,10)}$, cerebrovascular disease ${ }^{9.11)}$, and atherosclerotic risk factors $4,12,13)$. Actually, plaques in the extracranial carotid arteries are frequently found in and around the carotid bulbs ${ }^{14,15}$. However, as for the anatomic structure, the accuracy and reproducibility of the measurement of IMT in and around the bulbs is somewhat poorer than that measured in the parallel wall portion of the $\mathrm{CCA}^{2,3,16)}$. Moreover, some researchers believe that plaques tend to be located at specific artery sites, e.g. arterial bifurcation, and be influenced by the local anatomic features of the artery and nonlaminar turbulent blood stream, although atherosclerosis is a generalized disease ${ }^{3)}$.

On the other hand, thickening of the intima-media in the common carotid arteries is also considered to be an early marker of generalized atherosclerosis $2,5,16,17$. The IMT in the distal CCA starts to thicken early in the atherosclerotic process ${ }^{15}$, even though plaques are seen less frequently than in the bulbs ${ }^{1}$. An increased IMT may predict future atherosclerotic morbidity.

In contrast to the plaque of the bulbs, the relationships between IMT-CCA and atherosclerotic risk factors or diseases are somewhat weak ${ }^{3}$. However, IMT measurement in the par-

Department of Epidemiology, National Institute for Longevity Sciences.

Address for correspondence : Dr. Fujiko Ando, Department of Epidemiology, National Institute for Longevity Sciences, 36-3,
Gengo, Morioka-cho, Obu, Aichi, 474-8522 Japan. 
allel wall portion of the CCA has several advantages ${ }^{2}$. First, it is easier to visualize consistently the IMT there than in the bulbs because of the local structure ${ }^{2,18}$. Second, the reproducibility of IMT-CCA measurement is also higher than IMT in the bulbs (IMT-BLB) ${ }^{2,3,16,19)}$. Third, influences of local nonlaminar turbulent blood flow are less in the CCA than in the bifurcation ${ }^{3)}$. Hence, IMT-CCA measurement might be better for the assessment of general age-related changes in arteries in epidemiological studies ${ }^{1,2,3)}$.

However, the definition of the IMT-CCA in previous studies has not been uniform ${ }^{20}$. Some studies defined the IMT-CCA at the maximum point in the IMT ${ }^{2,3,21)}$ and others defined it as the mean of several fixed points ${ }^{22-24}$. With such measurements, IMT-CCA may include two quite different pathological changes. One is a general IMT change that involves the proliferation of cells and deposition of ground substance in the media. The other is a local atherosclerotic change which includes cell proliferation in the intima and deposition of alternated LDL-cholesterol, visualized as foam cell accumulation.

In the study of aging, it is very important to distinguish normal aging from pathogenic changes related to diseases ${ }^{25}$ ). Therefore, it might be better to estimate IMT-CCA without plaque than with plaque, even if the IMT-CCA change itself includes general pathogenic changes.

Moreover, the pathophysiological significance of general thickening of the intima-media with regard to the atherosclerotic process is not clear, although there were some reports about the relationship between IMT-CCA and plaque formation $14,17,26,27$. The major aim of this article was to assess IMTCCA at the thinnest point and local atherosclerosis in the bulbs, to investigate the mutual relationships among them and to clarify the effects of the local plaques on the relationship between IMT-CCA and age.

\section{MATERIALS AND METHODS}

\section{Subjects}

The subjects were 979 men and women who participated in the NILS-LSA (National Longevity Sciences- Longitudinal Study of Aging) from April, 1998 to March, 1999. The NILSLSA is a comprehensive longitudinal study of aging, which started in November, 1997. The participants were age- and gender-stratified random samples aged 40 to 79 years in the first wave examination from the NILS area. All participants gave their informed consent before they participated in the study. Details of the study purpose, design and examination procedures have been described elsewhere ${ }^{25}$. The study protocol was approved by the Committee of the Chubu National Hospital. This article is based on some of the baseline examination data.

\section{Measurements of IMT}

Carotid artery ultrasound scanning was carried out using an
Ultrasonic Tomography (EUB-655, Hitachi Medical Corp., Japan) with a $10 \mathrm{MHz}$ linear array transducer. The subjects were examined supine with the neck extended. Walls of the CCA were scanned longitudinally and transversely to assess the maximal and minimal points of IMT in the bulbs and the CCA. Then, all measurements of IMT were made in the longitudinal planes where the maximal or minimal IMT points could be well identified.

The IMT was defined as the distance between the inner echogenic line representing the intima-blood interface and the outer echogenic line representing the adventitia-media junction, as described by Pignoli et al. ${ }^{6}$ and others ${ }^{7,21)}$.

The position of the carotid bulbs was identified from the loss of the parallel wall in the CCA to the tip of the flow divider separating the external and internal carotid arteries. The maximum or minimum IMT in the bulbs and CCA within $1 \mathrm{~cm}$ proximal to the bulbs was defined as MAX-BLB or MIN$B L B$, because plaques are frequently present in and around the bulbs ${ }^{14)}$. However, as the measurement of IMT in the internal carotid artery is less reproducible ${ }^{2,3}$, it was not included in plaque assessments. In a similar way, the maximum or minimum IMT in the CCA from $1 \mathrm{~cm}$ proximal from the bulbs to the side of thymus was defined as MAX-CCA or MIN-CCA. The measurements were done in both the right and left sides.

At least 4 pictures were taken of each right and left CCA for measurement of IMT (MAX-BLB, MIN-BLB, MAX-CCA, MIN-CCA). After freezing the image, the measurement was done with electronic calipers. A 3-fold magnification of the ultrasound image was used to improve the accuracy of caliper placement.

\section{Intra- and inter-operator variability}

The intra- and inter-operator variability for repeated measurements of IMT with this technique was examined respectively in 32 and 62 subjects of the NILS-LSA, but only in the right carotid arteries.

\section{Analysis variables}

The many measurements of IMT were summarized into 3 variables, IMT-CCA, IMT-BLB and PLQ-BLB. The IMTCCA was defined as the mean of the MIN-CCA on both sides. The IMT-BLB was defined as the greater MAX-BLB on both sides. Plaques were defined as local thickening $(\geqq 1.1 \mathrm{~mm})$ of IMT ${ }^{1,4,28)}$. If IMT-BLB $\geqq 1.1 \mathrm{~mm}$, then PLQ-BLB was defined plus $(+)$, if not, then PLQ-BLB was minus $(-)$. Hence, PLQ-BLB (+) means at least one plaque exists in the bulbs. IMT-BLB or PLQ-BLB is a quantitative or qualitative plaque index.

\section{Statistical Analysis}

Data were analyzed with the Statistical Analysis System (SAS) release $6.12^{29)}$. All descriptive data are expressed as mean \pm standard deviation unless stated otherwise. A paired or 
unpaired, two-tailed Student's t test was used for comparisons of IMT measurements. When more than 2 groups were compared, this was done with analysis of variance. If the $F$ test demonstrated a significant difference among the groups, each pair of means was compared using Tukey's test. The age difference of IMT was also estimated with a trend test. CochranMantel-Haenszel (CMH) statistics were used for comparison of proportions. Univariate analyses of the associations between IMT and age and between IMT-CCA and IMT-BLB were performed with Pearson's correlation analysis. Interaction of IMT-CCA with age, gender, and plaque formation was then examined with multiple regression analysis. Statistical significance was inferred at $\mathrm{p}<0.05$.

\section{RESULTS}

\section{Intra- and inter-operator variability}

The results of the intra- and inter-operator variability assessment for repeated measurements of IMT are shown in Table 1. The correlation coefficients of intra-operator variability were 0.94-0.96 and the coefficients of variation $(\mathrm{CV})$ were 0.06 0.07 . The theoretical resolution of ultrasonography used in this study was $0.1 \mathrm{~mm}$ and the probability of a measurement difference $\leqq 0.1 \mathrm{~mm}$ was $0.97-1.00$. As for inter-operator variability, the correlation coefficients were $0.85-0.98$ and the $\mathrm{CV}$ were $0.06-0.09$. The probability of a measurement difference $\leqq 0.1$ mm was 0.89-0.94.

\section{The difference in IMT of the left and right CCA}

To assess local anatomical effects on the IMT measurements, the mean IMT difference between the left and right side $\left(\mathrm{IMT}_{\text {lefi }}-\mathrm{IMT}_{\text {right }}\right.$ ) was calculated (Table 2). There was no significant difference in MIN-CCA or MIN-BLB. On the other hand, the IMT was significantly larger on the left side com- pared to the right in the MAX-CCA or MAX-BLB (the differences were $0.041 \pm 0.009 \mathrm{~mm}$ (mean \pm standard error) and $0.028 \pm 0.005 \mathrm{~mm}$, respectively, $\mathbf{p}<0.0001$ ).

\section{Gender and age differences in the IMT and plaque formation}

The gender and age distributions of the subjects and IMT values are shown in Table 3. There was no significant difference in age distribution between men and women. In women, $350(72.9 \%)$ were postmenopausal. The mean value of the IMT-CCA in this population was $0.59 \pm 0.14 \mathrm{~mm}$. IMT-CCA was significantly higher in men than in women $(0.61 \pm 0.15$ $\mathrm{mm}$ and $0.58 \pm 0.14 \mathrm{~mm}$, respectively, $\mathrm{p}<0.01$ ). IMT-BLB was also significantly higher in men than in women $(1.19 \pm$ $0.60 \mathrm{~mm}$ and $1.08 \pm 0.48 \mathrm{~mm}$, respectively, $\mathrm{p}<0.01$ ). IMTCCA was $0.03 \mathrm{~mm}$, whereas IMT-BLB was $0.11 \mathrm{~mm}$, higher in men than in women. The gender difference was significantly greater in IMT-BLB than IMT-CCA ( $p<0.05)$.

As for plaque formation, the prevalence of PLQ-BLB $(+)$, i.e., the presence of at least one plaque in and around the bulbs at any side, also showed a gender difference (Table $3,52.3 \%$ in men and $41.5 \%$ in women, $\mathrm{p}<0.001$ ).

IMT-CCA and IMT-BLB increased with age in men and women as shown in Table 3 ( $p$ trend $<0.0001$ ). The prevalence of PLQ-BLB $(+)$ was only $25.4 \%$ in men and $17.8 \%$ in women in their $40 \mathrm{~s}$, but it increased to $79.5 \%$ and $71.1 \%$, respectively in the 70s age group. The $p$ trend was $<0.001$. The correlation coefficient of IMT-CCA with age $(r=0.517$, $p<0.0001)$ was significantly higher $(p<0.05)$ than that of IMT-BLB with age $(r=0.435, p<0.0001)$ (Table 4).

In univariate analysis considering IMT-CCA and age, the equations to predict the IMT-CCA were as follows;

(Men) IMT-CCA $(\mathrm{mm})=0.224+0.066 \times$ Age $/ 10$;

(Women) IMT-CCA $(\mathrm{mm})=0.198+0.065 \times$ Age $/ 10$;

As there was no statistically significant difference between

Table 1. Intra-and inter-operator variability for repeated IMT measurement.

\begin{tabular}{ccccc}
\hline & $\begin{array}{c}\text { correlation } \\
\text { coefficient } \\
(\mathrm{r})\end{array}$ & $\mathrm{p}$ value & $\begin{array}{c}\text { the prevalence } \\
\text { of the } \\
\text { difference } \leqq \\
0.1 \mathrm{~mm}\end{array}$ & $\begin{array}{c}\text { coefficient } \\
\text { of variation } \\
\text { (C.V) }\end{array}$ \\
\hline $\begin{array}{c}\text { Intra-assay (n=32) } \\
\text { MIN-CCA }\end{array}$ & 0.94 & $<0.0001$ & 1.00 & 0.06 \\
MAX-CCA & 0.96 & $<0.0001$ & 0.97 & 0.06 \\
MIN-BLB & 0.94 & $<0.0001$ & 1.00 & 0.07 \\
MAX-BLB & 0.96 & $<0.0001$ & 0.97 & 0.07 \\
& & & & \\
Inter-assay (n=62) & & & & 0.09 \\
MIN-CCA & 0.85 & $<0.0001$ & 0.94 & 0.07 \\
MAX-CCA & 0.96 & $<0.0001$ & 0.92 & 0.07 \\
MIN-BLB & 0.92 & $<0.0001$ & 0.92 & 0.06 \\
MAX-BLB & 0.98 & $<0.0001$ & 0.89 & \\
\hline The analyses were done only in the & & &
\end{tabular}

The analyses were done only in the right carotid arteries. 
Table 2. The difference between the left and right IMT-CCA.

\begin{tabular}{rccc}
\hline & \multicolumn{2}{c}{ IMT (left) - IMT (right) } \\
& mean & s.e. & $\mathrm{p}$ \\
\hline MIN-CCA & -0.001 & 0.016 & n.s. \\
MAX-CCA & 0.041 & 0.009 & $<0.0001$ \\
MIN-BLB & 0.012 & 0.008 & n.s. \\
MAX-BLB & 0.028 & 0.005 & $<0.0001$ \\
\hline & & \multicolumn{2}{c}{ n.s. : not significant }
\end{tabular}

Table 4. Correlation of IMT-CCA and IMT-BLB with Age.

\begin{tabular}{lccccc}
\hline & & \multicolumn{2}{c}{ IMT-CCA } & \multicolumn{2}{c}{ IMT-BLB } \\
& & r & p & r & p \\
\hline All & $(979)$ & 0.517 & 0.0001 & 0.435 & 0.0001 \\
Men & $(499)$ & 0.490 & 0.0001 & 0.427 & 0.0001 \\
Women (480) & 0.555 & 0.0001 & 0.454 & 0.0001 \\
\hline
\end{tabular}

The figures in parentheses showed the number of subjects.

Table 3. IMT-CCA, IMT-BLB and the prevalence of PLQ-BLB (+).

$\left.\begin{array}{ccllll}\hline \multicolumn{1}{c}{\text { Age }} & \text { Number } & \begin{array}{c}\text { Age } \\ \text { mean } \pm \text { s.d. }\end{array} & \begin{array}{c}\text { IMT-CCA } \\ \text { mean } \pm \text { s.d. }\end{array} & \begin{array}{c}\text { IMT-BLB } \\ \text { mean } \pm s . d .\end{array} & \begin{array}{c}\text { Prevalence of } \\ \text { PLQ-BLB (+) } \\ (\%)\end{array} \\ \hline \text { ALL } & 979 & 58.5 \pm 10.9 & 0.59 \pm 0.14 & 1.13 \pm 0.55 & 47.0 \\ \text { Men } & 499 & 58.4 \pm 11.0^{\mathrm{a}} & 0.61 \pm 0.15^{\mathrm{b}} & 1.19 \pm 0.60^{\mathrm{b}} & 52.3^{\mathrm{c}} \\ 40-49 & 138 & & 0.52 \pm 0.08 \\ 50-59 & 135 & & 0.59 \pm 0.12 \\ 60-69 & 104 & & 0.63 \pm 0.14 \\ 70-79 & 122 & & 0.70 \pm 0.18\end{array}\right)$ d $\left.\begin{array}{l}1.15 \pm 0.49 \\ 1.17 \pm 0.51\end{array}\right)$ d $\left.\begin{array}{c}54.1 \\ 53.8\end{array}\right) \mathrm{e}$

\footnotetext{
a ; There were no significant gender differences.

b; There was a significant gender difference $(p<0.01)$.

$c$; There was a significant gender difference $(p<0.001)$.

$\mathrm{d}$; The $\mathrm{p}$ trend was $<0.0001$.

$\mathrm{e}$; The $\mathrm{p}$ trend was $<0.001$.
}

the regression coefficients, the difference in gender was almost shown by the intercept coefficients. Hence, the thickening of IMT was about 4 years more advanced in men than in women and the IMT-CCA increased about $0.06-0.07 \mathrm{~mm}$ every 10 years in both genders.

Similarly, IMT-BLB was calculated from these equations;

(Men) IMT-BLB $(\mathrm{mm})=0.228+0.164 \times$ Age $/ 10$;

(Women) IMT-BLB $(\mathrm{mm})=0.104+0.166 \times$ Age $/ 10$;

There was also no significant difference in the regression coefficients. As the intercept was 0.124 higher in men than in women, the thickening of IMT-BLB in men was supposed to be about 7 or 8 years more advanced than in women.

\section{Relationship between IMT and local plaques}

Correlation coefficients between IMT-CCA and IMT-BLB were statistically significant $(r=0.492, p<0.0001)$. The IMTCCA in subjects without plaques was lower than in subjects with plaques $(0.53 \pm 0.10 \mathrm{~mm}$ and $0.66 \pm 0.15 \mathrm{~mm}$, respectively, $\mathrm{p}<0.0001$ ). Even if age and gender were adjusted for, the IMT-CCA in subjects without plaques was still significantly lower than in subjects with plaques $(0.55 \pm 0.12 \mathrm{~mm}$ and
$0.63 \pm 0.12 \mathrm{~mm}$, respectively, $\mathrm{p}<0.0001$ ).

Influence of local plaques on the relationship between IMT and age

Using a multiple regression model, the equations to predict IMT-CCA in subjects with or without plaques were as follows; PLQ-BLB $(+)$; IMT-CCA $(\mathrm{mm})=0.286+0.061 \times$ Age $110-$ $0.031 \times$ Gender;

PLQ-BLB $(-)$; MT-CCA $(\mathrm{mm})=0.319+0.041 \times$ Age $/ 10-$ $0.015 \times$ Gender;

(Gender; Men $=0$, Women $=1$ )

We thus found the IMT-CCA in subjects with plaques increased $0.06 \mathrm{~mm}$ every 10 years, whereas that without plaques increased only $0.04 \mathrm{~mm}$ every 10 years.

If PLQ-BLB was treated as an explanatory variable like age and gender in a multiple regression model, these variables explained approximately $31.7 \%$ of IMT variance. The relative contribution of each variable is shown in Table 5 . 


\section{DISUCUSSION}

The carotid arteries have been of particular interest to researchers because they are easily and noninvasively accessible using ultrasonography. Quantitative measurement of IMT performed in the distal segment of the CCA just proximal to the carotid bulbs has come to be equated with atherosclerosis, mostly because this variable is associated with cardiovascular disease ${ }^{1,3,30)}$, its risk factors ${ }^{3,12,13)}$ and other peripheral atherosclerotic diseases 3,26 ).

As plaque formation is frequently seen in that site of $\mathrm{CCA}^{1,3)}$, however, the measurement of IMT may include two quite different pathophysiological changes. One is a general age-related change in the intima-media complex, which involves the diffuse thickening of media due to progressive smooth muscle proliferation and ground substance accumulation ${ }^{31}$ ) It is recognized early in the atherosclerotic process $2,5,16,17$. The other change is a local atherosclerotic change, such as atherosclerotic streak and plaque formation through a process linked to lipid accumulation. These pathological findings are mostly recognized as thickening of the inti$\mathrm{ma}^{3}$.

Moreover, the definitions of IMT in previous studies were miscellaneous ${ }^{20)}$, such as the single value at the maximal point ${ }^{5,32}$, the mean of several measurements of the maximal points ${ }^{2,3,33)}$, or the mean at several fixed points or lengths ${ }^{4,34)}$. Therefore, the value of IMT in these studies varied 0.4-1.0 $\mathrm{mm}$, while the progression rate also ranged widely from 0.01 to $0.3 \mathrm{~mm} /$ year $^{20)}$.

The definition of IMT-CCA in this study was the mean value of the measurements at the thinnest points on both sides of the CCA, from just $1 \mathrm{~cm}$ proximal to the bulbs to the side of the thyroid. This definition has several advantages. First, as the consistency and reproducibility of the measurement in this site is excellent ${ }^{2,3)}$, it would be appropriate for epidemiological study. Second, plaques could avoid measurement because of the selection of the thinnest point of IMT. Moreover, local influence of nonlaminar turbulent blood flow might be slight, because the CCA is straight and nonbranching in the site ${ }^{2,33}$. Hence, IMT-CCA measurement in our method might be better than previous methods for the assessment of general age-related changes in IMT-CCA in epidemiological studies.

In this study, we focused our attention on the thickness of the intima-media complex at the thinnest point in the CCA. The aim of this study was to investigate its gender difference, agerelated changes and the relationship with the presence of atherosclerotic plaques in carotid bifurcation.

Compared other studies ${ }^{1,2,4)}$, the results of intra- and interoperator assays were sufficient to do further analysis.

There was a significant difference between IMT on the left and right sides of both MAX-CCA and MAX-BLB, which included more atherosclerotic pathological change than MINCCA and MIN-BLB. On the other hand, there was no significant side difference in MIN-CCA and MIN-BLB, which represented general thickening of the intima-media. A side difference between IMT on the left and right sides was described earlier ${ }^{35-37}$, strongly suggesting that this was not a chance finding. One proposed explanation has been that atherosclerosis develops faster on the left side because of anatomic differences between the sides and thus different shear stress conditions ${ }^{35}$. Our results did not contradict these previous studies.

The mean value of the IMT-CCA in our study was somewhat lower than that in previous studies ${ }^{1,20)}$. This may be due to the site of measurement, measurement procedure, population characteristics or the ethnic group. In Japan, there have been quite few population-based studies on IMT-CCA references on a large scale. Yamasaki et al reported IMT-CCA among healthy men $(n=55)$ was $0.72 \pm 0.03,0.82 \pm 0.02$, $0.87 \pm 0.09 \mathrm{~mm}$ in the $40 \mathrm{~s}, 50 \mathrm{~s}$, and $60 \mathrm{~s}$ and over ${ }^{38}$. Although their subjects were apparent healthy persons without diabetes, hyperlipidemia, or other risk factors of atherosclerosis, the values were higher than those in our study. This may be partially due to the measurement method, because they defined IMT as the average of three points; one was the thickest point in the CCA and other two points were $1 \mathrm{~cm}$ upstream and $1 \mathrm{~cm}$ downstream from the thickest point.

The discrepancy between men and women in the present study could be due to the fact that men develop atherosclerosis at an earlier age than women. IMT was generally higher in men than in women. The same result was also found in other studies

Table 5. Summary of the multivariate model for prediction of IMT-CCA.

\begin{tabular}{lccccc}
\hline Variable & $\beta 1$ & SE1 & $\beta 2$ & SE2 & p \\
\hline Age & 0.005 & 0.0003 & 0.383 & 0.029 & $<0.0001$ \\
Gender & -0.022 & 0.008 & -0.076 & 0.026 & $<0.01$ \\
PLQ-BLB & 0.079 & 0.008 & 0.275 & 0.029 & $<0.0001$ \\
\hline
\end{tabular}

Adjusted multiple $\mathrm{R}^{2}=0.31$

$\beta 1$ is the raw regression coefficient and SEl is the standard error of $\beta 1$.

$\beta 2$ is the standardized regression coefficient and SE2 is the standard error of $\beta 2$.

Gender; Men=0, Women=1

PLQ-BLB; PLQ-BLB(-) $=0, \operatorname{PLQ}-B L B(+)=1$ 
of similar design in the 60 to 70 years old age group ${ }^{7.37,39)}$.

Although this study was a cross-sectional study, we tried to predict gender differences in the age-related progression of thickening in the intima-media complex. As there was no statistically significant difference in the regression coefficients of the equations of IMT-CCA between the two genders, agerelated changes were thought to be similar among men and women in this population, although the change in men was assumed to be several years more advanced than that in women. Allan et al, also suggested that levels of atherosclerotic development in the common carotid artery are 5 to 10 years more advanced in men than in women ${ }^{37}$, but they did not mention gender differences in the speed of thickening. It is well known that there is a marked gender difference in the progression of atherosclerotic lesions. There was also no statistically significant difference in the regression coefficients of MT-BLB between the two genders. As IMT-BLB is supposed to be a quantitative index for local atherosclerotic changes, not only the progression speed of IMT but also that of carotid atherosclerosis, might be similar between both genders in this middle-aged and old population. The high ratio of postmenopausal women $(72.9 \%)$ in this population may have influenced on the results. To exclude the influence of menstruation and early menopause due to ovarian surgery, the equation for women aged 56 and over was calculated as follows;

$\operatorname{IMT}$-CCA $(\mathrm{mm})=0.197+0.065 \times$ Age $/ 10$;

The intercept and regression coefficient were similar to the equation for all women. Further research on the gender difference in IMT-CCA should take into account not only menstruation and early menopause due to ovarian surgery, but also hormone replacement therapy, the gender difference in the prevalence of smoking, hypertension, obesity and other gender-related atherogenic factors.

IMT-CCA and IMT-BLB increased with age and the correlation coefficient of IMT-CCA with age was higher than that of IMT-BLB. General thickening of the intima-media complex may be more age-related than local atherosclerotic changes.

In this study, IMT-CCA was assumed to increase about 0.07 mm every 10 years and the value was remarkably low 1 . Kanters et al studied 23 articles ${ }^{20)}$, which included descriptions of the reliability of IMT-CCA measurement. The progression rate of IMT ranged from 0.01 to $0.3 \mathrm{~mm} /$ year in these articles. But many of the subjects in these studies were patients and the IMT measured included local atherosclerotic changes. IMTBLB increased $0.16 \mathrm{~mm} / 10$ year in our study and this might be compatible with the results of previous studies.

IMT-CCA was positively related to the presence and severity of the local plaques in this study, as found by others ${ }^{17,27,40 \text { ). }}$ IMT-CCA in subjects with plaques increased $0.06 \mathrm{~mm}$ every 10 years, whereas those without plaques increased only 0.04 $\mathrm{mm}$ every 10 years. This implies that local plaque formation itself or the risk factors related to local plaque formation influence the progression rate of IMT.
Finally, age, gender and local plaques in the bifurcation explained $31.7 \%$ of IMT-CCA, but gender had a relatively small effect on IMT-CCA. In conclusion, even when the influences of local atherosclerosis were excluded as much as possible from IMT-CCA measurement, IMT-CCA was related moderately to age and local plaque formation. Probably, the atherosclerotic risk factors related not only to plaque formation, but also the thickening of the IMT. Both longitudinal study and multivariate analysis, which includes atherosclerotic risk factors and atherosclerotic diseases, are needed to understand better the natural history and risk factors intima-media thickening and how IMT influences the incidence and severity of atherosclerotic diseases.

\section{ACKNOWLEDGEMENTS}

We thank all of the participants and colleagues in the NILSLSA. This study was supported in part by a Grant-in-Aid for Comprehensive Research on Aging and Health from the Ministry of Health and Welfare of Japan.

\section{REFERENCES}

1. Rosfors $S$, Hallestam S, Jensen-Urstad K, Zetterling $M$, Carlstrom $\mathrm{C}$. Relationship between intima-media thickness in the common carotid artery and atherosclerosis in the carotid bifurcation. Stroke, 1998;29:1378-82.

2. Sidhu PS, Desai SR. A simple and reproducible method for assessing intima-media thickness of the common carotid artery. British Journal of Radiology,1997;70:8589.

3. O'Leary DH, Polak JF, Kronmal RA, et al. Thickening of the carotid wall-a marker for atherosclerosis in the elderly-. Stroke, 1996;27:224-231.

4. Bonithon-Kopp C, Touboul PJ, Berr C et al. Relation of intima-media thickness to atherosclerotic plaques in carotid arteries. The Vascular Aging (EVA) Study. Arteioscler Thromb Vasc Biol, 1996;16:310-316.

5. O'Leary DH, Polak JF, Kronmal RA, et al.Carotid-artery intima and media thickness as a risk factor for myocardial infarction and stroke in older adults. N Engl J Med, 1999;340:14-22.

6. Pignoli P, Tremoli E, Poli A, Paoletti R. Intimal plus medial thickness of the arteril wall: a direct measurements with ultrasond imaging. Circulation, 1986;74:1399-1406.

7. Adams MR, Nakagomi A, Keech A, Robinson J, McCredie R, Bailey BP. Carotid intima-media thickness is only weakly correlated with the extent and severity of coronary artery disease. Circulation, 1995;92:2127-2134.

8. Probsfield JL, Byington RP, Egan DA, et al. Methodological issues facing studies of atherosclerotic change. Circulation. 1993;87(Suppl. II ):74-81.

9. Cave EM, Pugh ND, Wilson RJ, Sissons GRJ, Wood 
cock JP. Carotid artery duplex scanning does plaque echogenicity correlate with patinets symptoms? Eur $\mathbf{J}$ Vasc Endvasc Surg. 1995; 10:77-81.

10. European Carotid Plaque Study Group. Carotid artery plaque composition: relationship to clinical presentation and ultrasound B-mode imaging. Eur J Vasc Endvasc Surg, 1995;10:23-30.

11. Steinke W, Hennerici M, Rautenberg W, Mohr JP. Symptomatic and asymptomatic high-grade carotid stenosis in Doppler color-flow imaging. Neurology, 1992;42:131-138.

12. Tell GS, Howard G, McKinney WM. Risk factors for site specific extracranial carotid artery plaque distribution as measured by B-mode ultrasound. J Clin Epidemiol, 1989:42:551-559.

13. Poli A, Tremoli E, Colombo A et al. Ultrasonographic measurements of the common carotid artery wall thickness in hypercholesterolemic patients. A new model for the quantitaion and follow-up of preclinical atherosclerosis in living human subjects. Atherosclerosis, 1988;70:253-261.

14. Riley WA, Barnes RW, Applegate WB, et al. Reproducibility of noninvasive ultrasonic measurement of carotid atherosclerosis. Stroke, 1992;23:1062-1068.

15. Martin MJ, Whisnant JP, Sayre GP. Occlusive vascular disease in extracranial cerebral circulaion. Arch Neurol 1960;5:64-72.

16. Bonithon-Kopp C, Coudray C, Berr C, et al. Combined effects of lipid peroxidation and antioxidant status on carotid atherosclerosis in a population aged 59-71:The EVA Study. Am J Clin Nutr, 1997;65:121-127.

17. Persson J, Formgren J, Israelsson B, Berglund G. Ultrasound determined intima-media thickness and atherosclerosis: direct and indirect validation. Arterioscler Thromb, 1994;14:261-264.

18. Howard G, Sharrett AR, Heiss G, et al. Carotid artery intimal-medial thickness: distribution in general populations as evaluated by B-mode ultrasound. Stroke, 1993;24:1297-1304.

19. Salonen JT., Salonen R.Association of serum low density lipoprotein cholesterol, smoking and hypertension with different manifestations of atherosclerosis. Int $\mathbf{J}$ Epidemiol.1990;19:911-917.

20. Kanters SDJM, Algra A, Leeuwen MS, Banga JD. Reproducibility of in vivo carotid intima-media thickness measurements. Stroke, 1997;28:665-671.

21. Salonen R, Salonen JT. Progression of carotid atherosclerosis and its determinants: a population-based ultrasonography study. Atherosclerosis, 1990;81:33-40.

22. Schmidt-Trucksass A, Grathwohl, Schmid A, Boragk R, Upmeier C, Keul J Huonker M. Structural, functional, and hemodynamic changes of common carotid artery with age in male subjects. Arterioscler Thromb Vasc
Biol, 1999;19:1091-1097.

23. Nagai Y, Metter EJ Earley C, et al. Increased carotid artery intima-media thickness in asymptomatic older subjects with exercise-induced myocardial ischemia. Circulation, 1998:98:1504-1509

24. Cerhan JR, Folsom AR, Mortimer JA, et al. Correlates of cognitive function in middle-aged adults. Gerontology, 1998;44:95-105.

25. Shimokata $H$, Ando F, Niino N. A new comprehensive study on aging-National Institute for Longevity Sciences, Longitudinal Study of Aging (NILS-LSA). J Epidemiology, 2000;10:S1-S9.

26. Veller MG, Fisher CM, Nicolaides AN, et al. Measurement of the ultrasonic intima-media complex thickness in normal subjects. J Vasc Surg, 1993;17:19-25.

27. Wendelhag I, Wiklund O, Wikstrand J. Atherosclerotic changes in the femoral and carotid arteries in familial hypercholesterolemia. Ultrasonographic assessment of intima-media thickness and plaque occurrence. Arterioscler Thromb, 1993;13:1404-11.

28. Wendelhag I, Wiklund O, Wikstrand J. Arterial wall thickness in familial hypercholesteremia: ultrasound measurement of intima-media thickness in the common carotid artery. Athroscler Thromb, 1992;12:70-77.

29. SAS Procedure Guide, Release 6.12 Edition. SAS Institute Inc., Cary, NC, 1997.

30. Salonen JT., Salonen R. Ultrasonographically assessed carotid morphology and the risk of coronary heart disease. Arterioscler Thromb, 1991;11:1245-1249.

31. Ross R. The pathogenesis of atherosclerosis:an update. $\mathbf{N}$ Engl J Med, 1986;314:488-500.

32. O'Leary DH, Polak JF, Wolfson SK Jr, et al. Use of sonography to evaluate carotid atherosclerosis in the elderly: the Cardiovascular Health Study. Steoke, 1991;22:11551163.

33. Sharrett AR, Sorlie PD, Chambless LE et al. Relative importance of various risk factors for asymptomatic carotid atherosclerosis versus coronary heart disease incidence. The atherosclerosis risk in communities study. Am J Epidemiol, 1999;149:843-52.

34. Bots ML, Jong PTVM, Hofman A, Grobbe DE. Left, right, near or far wall common carotid Intima-media thickness measurements: associations with cardiovascular disease and lower extremity arterial atherosclerosis. J Clin Epidemiol, 1997;50:801-807.

35. Lemne C, Jogestrand T, de Faire U. Carotid intima-media thickness and plaques in borderline hypertension. Stroke, 1995;26,34-39.

36. Jensen-Urstad KJ, Reichard PG, Rosfors JS, Lindblad LEL, Jensen-Urstad MT. Early atherosclerosis is retarded by improved long-term blood glucose control in patients with IDDM. Diabetes, 1996;45:1253-1258.

37. Allan PL, Mowbray PI, Lee AJ, Fowkes GR. Relationship 
between carotid - ntima-media thickeness and symptomatic and asymptomatic peripheral arterial diseas. Stroke, 1997;28:348-353.

38. Yamasaki $\mathrm{Y}$, Kawasaki R, Matsushima $\mathrm{H}$ et al. Asymptomatic hyperglycemia is associated with increased intimal plus medial thickness of the carotid artery. Diabetologia, 1995;38:585-591.

39. Bonithon-Kopp C, Touboul J, Berr C, Magne C,
Ducimetiere P. Factors of carotid arterial enlargement in a population aged 59 to 71 years. The EVA Study. Stroke, 1996;27:654-660.

40. Wofford JL, Kahl FR, Mckinney WM, Toole JF, Crouse JR III. Relation of extent of extracarotid artery atherosclerosis as measured by B-mode ultrasound to the extent of coronary atherosclerosis. Arterioscler. Thromb. 1991;11:1786-1794. 